\title{
Hole Subband Mixing and Polarization of Luminescence from Quantum Dashes: A Simple Model
}

\author{
P. Kaczmarkiewicz, A. Musiat, G. Sęk, P. Podemski, P. Machnikowski \\ AND J. MISIEWICZ \\ Institute of Physics, Wrocław University of Technology \\ Wybrzeże Wyspiańskiego 27, 50-370 Wrocław, Poland
}

\begin{abstract}
In this paper, we address the problem of luminescence polarization in the case of nanostructures characterized by an in-plane shape asymmetry. We develop a simple semi-qualitative model revealing the mechanism that accounts for the selective polarization properties of such structures. It shows that they are not a straightforward consequence of the geometry but are related to it via valence subband mixing. Our model allows us to predict the degree of polarization dependence on the in-plane dimensions of investigated structures assuming a predominantly heavy hole character of the valence band states, simplifying the shape of confining potential and neglecting the influence of the out-of-plane dimension. The energy dependence modeling reveals the importance of different excited states in subsequent spectral ranges leading to non-monotonic character of the degree of polarization. The modeling results show good agreement with the experimental data for an ensemble of InAs/InP quantum dashes for a set of realistic parameters with the heavy-light hole states separation being the only adjustable one. All characteristic features are reproduced in the framework of the proposed model and their origin can be well explained and understood. We also make some further predictions about the influence of both the internal characteristics of the nanostructures (e.g. height) and the external conditions (excitation power, temperature) on the overall degree of polarization.
\end{abstract}

PACS: 78.67.Hc, 73.22.-f

\section{Introduction}

Quantum dashes (QDashes) [1-6] are quasi-zero-dimensional nanostructures characterized by strong in-plane asymmetry. Typically, their width is on the order of several to a few tens of nanometers, while their length may be on the order of hundreds of nanometers [1-4]. The electronic structure and optical properties of these structures have been investigated both experimentally [4-8] and theoretically [7, 9-11], which is motivated by their favorable properties from the point of view of optoelectronic applications at telecom wave lengths [5]. For those and other applications, the understanding of polarization properties (expected to be anisotropic due to shape asymmetry) is crucial.

In Fig. 1a we show the polarization-dependent photoluminescence spectrum obtained at $300 \mathrm{~K}$ from an ensemble of epitaxially grown self-assembled InAs/InP QDashes preferentially elongated in the [110] direction, similar to those studied in Ref. [2, 4]. Figure 1b shows the corresponding degree of polarization (DOP),

$$
P=\frac{I_{\|}-I_{\perp}}{I_{\|}+I_{\perp}},
$$

where $I_{\|}, I_{\perp}$ are the intensities of luminescence components polarized along and perpendicular to the QDash elongation axis. The anisotropic shape of the QDashes
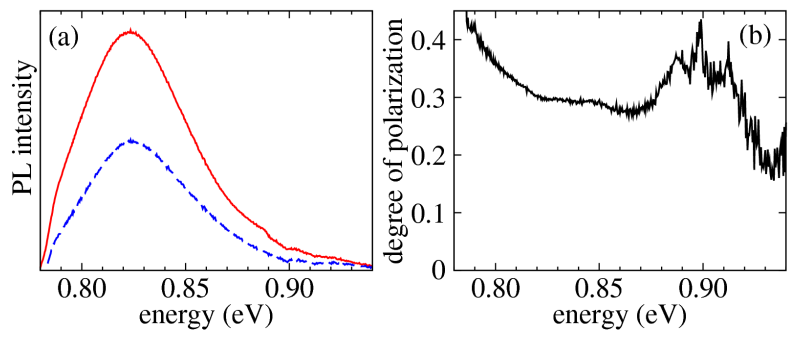

Fig. 1. (a) Room temperature photoluminescence spectra of an ensemble of QDashes at two orthogonal linear polarizations (red solid line: parallel to the structure elongation, blue dashed line: perpendicular to the structure elongation). (b) The corresponding degree of polarization. The apparent structure in the high-energy part results from irrelevant fluctuations due to water vapor absorption in the area of weak photoluminescence signal.

leads to a high DOP of the emitted radiation (see also Ref. [8]). This polarization cannot be a trivial consequence of the confinement shape at the level of a single-band effective mass and envelope function description as the emission is governed by the interband dipole moment that depends on the Bloch parts of the wave functions and not on their envelopes. Therefore, a more gen- 
eral description is needed in order to take subband mixing into account. Quantitatively exact modeling of such non-trivial optical properties of nanostructures must be based on atomistic or multi-band $k p$ methods [10, 12]. However, it may be useful and interesting to have a simple semi-quantitative model that would not only elucidate the physical mechanism of the polarization but also yield an estimate of the expected effect in terms of the shape parameters.

In this contribution, we present a "minimal" theory that is able to account for the observed DOP in the QDash luminescence (a similar model for trion transitions was proposed in Ref. [13]). First, we show that the polarization is proportional to the degree of heavy-light hole mixing. Then we estimate the degree of the latter within a semi-quantitative model and show that it is related to the shape asymmetry of the structure. We provide a very simple formula relating the DOP to the QDash dimensions.

\section{The model}

The polarization of light emitted in a recombination process from a conduction band $(\mathrm{cb})$ state $\Psi_{\mathrm{c}}(\boldsymbol{r})$ to a valence band (vb) state $\Psi_{\mathrm{v}}(\boldsymbol{r})$ is determined by the interband matrix element of the dipole moment operator $\hat{\boldsymbol{d}}$,

$$
\boldsymbol{d}=\int \mathrm{d}^{3} r \Psi_{v}^{*}(\boldsymbol{r}) \hat{\boldsymbol{d}} \Psi_{\mathrm{c}}(\boldsymbol{r})=\sum_{\lambda} a_{\lambda} \boldsymbol{d}_{\lambda} .
$$

Here we have performed the standard separation of length scales in the integration, $\boldsymbol{d}_{\lambda}$ is the bulk interband dipole moment between the initial cb (say, with spin up) and the final valence subband, and

$$
a_{\lambda}=\int \mathrm{d}^{3} r \psi_{\lambda}^{*}(\boldsymbol{r}) \psi_{\mathrm{c}}(\boldsymbol{r})
$$

is the envelope function overlap, where we denoted the cb envelope wave function by $\psi_{\mathrm{c}}(\boldsymbol{r})$ and the components of the vb envelope by $\psi_{\lambda}(\boldsymbol{r})$, where $\lambda$ is the subband index.

For the initial state in the spin-up conduction subband, only two transitions yield non-vanishing in-plane components of the dipole moment: to the $+3 / 2$ heavy hole band and to the $-1 / 2$ light hole band. The corresponding bulk matrix elements are

$$
\boldsymbol{d}_{3 / 2}=\frac{d_{0}}{\sqrt{2}}\left(\begin{array}{r}
-1 \\
\mathrm{i}
\end{array}\right), \quad \boldsymbol{d}_{-1 / 2}=\frac{d_{0}}{\sqrt{6}}\left(\begin{array}{l}
1 \\
\mathrm{i}
\end{array}\right),
$$

where the two components of the in-plane vectors refer to the Cartesian coordinates $x, y$ bound to the crystallographic axes.

In order to study the properties of a nanostructure elongated along the $[1 \overline{1} 0]$ direction we define the unit vectors along and transverse to the elongation direction

$$
\hat{\boldsymbol{e}}_{1}=\frac{\hat{\boldsymbol{e}}_{x}-\hat{\boldsymbol{e}}_{y}}{\sqrt{2}}, \quad \hat{\boldsymbol{e}}_{\mathrm{t}}=\frac{\hat{\boldsymbol{e}}_{x}+\hat{\boldsymbol{e}}_{y}}{\sqrt{2}} .
$$

The components of the interband dipole moment along these directions are

$$
d_{1}=-d_{0} \frac{\mathrm{i}+1}{2} a_{3 / 2}+d_{0} \frac{1-\mathrm{i}}{2 \sqrt{3}} a_{-1 / 2}
$$

and

$$
d_{\mathrm{t}}=d_{0} \frac{\mathrm{i}-1}{2} a_{3 / 2}+d_{0} \frac{\mathrm{i}+1}{2 \sqrt{3}} a_{-1 / 2} .
$$

Let us note that we use the standard definition of the basis functions $[14,15]$ (which differs from that used in many papers employing the $k p$ theory $[12,16])$.

The intensities of the linearly polarized components of the emitted radiation along and transverse to the structure are proportional to $\left|d_{\mathrm{l}}\right|^{2}$ and $\left|d_{\mathrm{t}}\right|^{2}$, respectively. Hence, the DOP is $P=\left(\left|d_{l}\right|^{2}-\left|d_{\mathrm{t}}\right|^{2}\right) /\left(\left|d_{\mathrm{l}}\right|^{2}+\left|d_{\mathrm{t}}\right|^{2}\right)$. From Eqs. (1) and (2), one finds

$$
\left|d_{1}\right|^{2}+\left|d_{\mathrm{t}}\right|^{2}=\left|d_{0}\right|^{2}\left[\left|a_{3 / 2}\right|^{2}+\frac{1}{3}\left|a_{-1 / 2}\right|^{2}\right]
$$

and

$$
\left|d_{1}\right|^{2}-\left|d_{\mathrm{t}}\right|^{2}=-\frac{2}{\sqrt{3}}\left|d_{0}\right|^{2} \operatorname{Im}\left[a_{3 / 2}^{*} a_{-1 / 2}\right] .
$$

From these equations, it is clear that mixing between heavy and light hole contributions in the confined hole state can lead to anisotropy of emission polarization with respect to the structure geometry, depending on the relative phase of the light-hole and heavy-hole components.

A more quantitative conclusion may be achieved if one assumes that the lowest hole state is predominantly of heavy hole character, with an admixture of light hole states. This is justified in many structures since the light hole states are shifted in energy with respect to the heavy hole states due to confinement and positive biaxial strain and the interband coupling elements are relatively small. It follows from the structure of the Kane Hamiltonian $[15,16]$ that the heavy hole state with the angular momentum $+3 / 2$ is coupled in the leading order of perturbation to both light hole states. However, the coupling term between this heavy hole state and the $+1 / 2$ state is proportional to $k_{z}$ which means that only states excited along the $z$ direction are coupled. Due to the strong confinement in the growth direction these states have a very high energy and their contribution is expected to be small. Thus, the hole state is essentially composed of the $+3 / 2$ hh component with an admixture of $\mathrm{a}-1 / 2 \mathrm{lh}$ component. In the 1 st order of perturbation theory one finds for the lh admixture

$$
\begin{aligned}
& \psi_{-1 / 2}(\boldsymbol{r})=\sum_{n} \frac{1}{\Delta E_{\mathrm{lh}}} \int \mathrm{d}^{3} r^{\prime} \phi_{n}^{*}\left(\boldsymbol{r}^{\prime}\right) V \psi_{3 / 2}\left(\boldsymbol{r}^{\prime}\right) \phi_{n}(\boldsymbol{r}) \\
& =\frac{1}{\Delta E_{\mathrm{lh}}} V \psi_{3 / 2}(\boldsymbol{r})
\end{aligned}
$$

where $\left\{\phi_{n}\right\}$ is any complete set of functions, $V$ is the relevant inter-subband element of the $k p$ Hamiltonian, $\Delta E_{\mathrm{lh}}=E_{\mathrm{h}}-E_{\mathrm{l}}$ is the energy separation between the heavy and light hole subbands, the differences between energies of various lh states have been neglected, and the last equality is obtained from the completeness relation 


$$
\sum_{n} \phi_{n}^{*}\left(\boldsymbol{r}^{\prime}\right) \phi_{n}(\boldsymbol{r})=\delta\left(\boldsymbol{r}-\boldsymbol{r}^{\prime}\right) .
$$

With our choice of basis states, the element of the Kane Hamiltonian coupling the relevant states is [15]

$$
V=\frac{\sqrt{3} \hbar^{2}}{2 m_{0}}\left[\gamma_{2}\left(k_{x}^{2}-k_{y}^{2}\right)+2 \mathrm{i} \gamma_{3} k_{x} k_{y}\right]
$$

where $k_{j}=\mathrm{i} \partial / \partial x_{j}, m_{0}$ is the free electron mass and $\gamma_{j}$ are the Luttinger parameters. Only the imaginary part of $V$ contributes to $\operatorname{Im}\left[a_{3 / 2}^{*} a_{-1 / 2}\right]$,

$$
\operatorname{Im} V=-\frac{\sqrt{3} \hbar^{2}}{2 m_{0}} \gamma_{3}\left(k_{1}^{2}-k_{\mathrm{t}}^{2}\right),
$$

where we used the components relative to the structure elongation.

In order to obtain a general estimate without relying on specific information on the confined wave functions we note that the dominating contribution to luminescence originates from electron and hole wave functions with the same quantum numbers and neglect the differences between their exact shape, so that $a_{3 / 2} \approx 1$. Assuming the simplest rectangular box model for the confinement we find

$$
\begin{gathered}
\operatorname{Im} a_{-1 / 2}=\frac{1}{\Delta E_{l h}} \int \mathrm{d}^{3} r \psi_{\mathrm{c}}(\boldsymbol{r}) V^{*} \psi_{3 / 2}^{*}(\boldsymbol{r}) \\
\quad \approx-\frac{\sqrt{3} \hbar^{2} \pi^{2}}{2 m_{0} \Delta E_{l h}} \gamma_{3}\left(\frac{n_{\mathrm{t}}^{2}}{D^{2}}-\frac{n_{1}^{2}}{L^{2}}\right),
\end{gathered}
$$

where $L, D$ are the dash length and width, respectively and $n_{\mathrm{l}}, n_{\mathrm{t}}$ are the corresponding quantum numbers in the rectangular box model. Hence, the DOP is

$$
P=\frac{\hbar^{2} \pi^{2}}{m_{0} \Delta E_{l h}} \gamma_{3}\left(\frac{n_{\mathrm{t}}^{2}}{D^{2}}-\frac{n_{1}^{2}}{L^{2}}\right) \text {. }
$$

\section{Results and discussion}

The formula (3) is the main theoretical result of our study. In spite of its simplicity and approximate character, it reproduces experimental data reasonably, as can be seen by comparing the measured DOP shown in Fig. 1 with the results of theoretical modeling presented in Fig. 2. Here, we plot the results obtained from an ensemble of QDashes modeled by a set of boxes (with infinite potential walls) characterized by the fixed height to width ratio of $H / D=1 / 3$, identical length $L=150 \mathrm{~nm}$ and a Gaussian distribution of ground state energies with the standard deviation of $44 \mathrm{meV}$ (which uniquely determines the size distribution). This set of parameters corresponds to the geometry of the QDash ensemble $[2,4]$ and to the inhomogeneous broadening of the low temperature photoluminescence spectra (not shown here). We have chosen the effective masses of electrons and holes to be $m_{\mathrm{e}}=0.07 m_{0}$ and $m_{\mathrm{h}}=0.3 m_{0}$, respectively, where $m_{0}$ is the free electron mass, which yields the lowest optical transition corresponding to a bright state excited in the transverse direction $\left(n_{1}=1, n_{\mathrm{t}}=2\right)$ at the energy $\Delta E=41 \mathrm{meV}$ above the ground state. Fitting to the overall polarization value observed in the experiment yields the heavy-light hole separation $\Delta E_{\mathrm{lh}}=30 \mathrm{meV}$, which is a reasonable value (actually, however, this energy should be correlated with the dot height, which we neglect). The results correspond to the thermal distribution of electron and hole occupations at $300 \mathrm{~K}$, assuming weak enough excitation to keep the Fermi system non-degenerate.
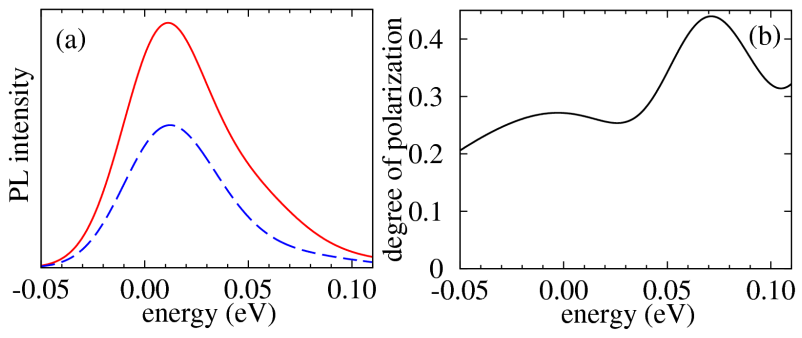

Fig. 2. (a) Theoretical results for the room temperature photoluminescence spectra of an ensemble of QDashes at two orthogonal linear polarizations (red solid line: parallel to the structure elongation, blue dashed line: perpendicular to the structure elongation). (b) The corresponding degree of polarization. The energies are shown relative to the ground state at the maximum of the distribution.

While the overall DOP follows from the value of the heavy-light hole energy splitting which is a fitting parameter, the agreement of the features appearing in the energy dependence is remarkable. Both in the theory and experiment, the DOP is roughly constant in the area of the maximum luminescence $(820 \mathrm{meV}$ in the experimental results). Slightly to the higher energies, the DOP drops. This can be understood from Eq. (3). At higher energies, the states excited along the structure $\left(n_{1}>1\right)$ have a larger contribution, which reduces the polarization. Further towards the higher energies, the state excited perpendicular to the structure $\left(n_{\mathrm{t}}=2\right)$ starts to contribute strongly. At $300 \mathrm{~K}$, the occupation of this state is non-negligible. According to Eq. (3), for low values of $n_{1}$, the DOP of the luminescence from this state is four times higher than that of the ground state. This leads to a clear maximum in the DOP observed in the experiment and reproduced in the model. On the low energy side of the luminescence maximum, the theory predicts a decrease in the DOP due to the increasing contribution of larger dots, while the ground state emission dominates. The discrepancy with respect to the experimental value is due to the abrupt decrease of the detection sensitivity below $0.78 \mathrm{eV}$ (InGaAs detector) causing the increased uncertainty of the experimental results in the area of weak luminescence signal.

In general, Eq. (3) yields many predictions for the DOP dependence on various system parameters. For structures with a small asymmetry $(D \sim L)$, the DOP should increase with growing $L$. However, this increase saturates and for strongly elongated structures $(L \gg D)$ 
at low temperatures and weak excitations, where the ground state emission dominates, one finds $P \sim 1 / D^{2}$, independent of $L$. The DOP should decrease and then increase with both temperature and excitation power as both these factors lead to an increased contribution from the excited states, first along the structure (which decreases the DOP), then transverse to the structure (which increases the DOP). Finally, semi-speculatively and beyond the presented formal model, one can expect that higher structures (larger $H$ ) should generally have smaller heavy-light hole separations and therefore should show stronger polarization.

\section{Conclusion}

In conclusion, we have formulated a semi-quantitative model that explains the polarization of light emitted by a nanostructure with a strong in-plane asymmetry (a quantum dash) by relating it to the hole subband mixing. The model yields a simple estimate of the DOP in terms of the geometrical and material parameters. We have tested our model against a room temperature measurement performed on an ensemble of InAs/InP QDashes. We have shown that the model not only accounts for the overall DOP for a reasonable value of the assumed heavy-hole splitting but also reproduces the features observed in the energy dependence of the DOP and allows us to interpret them in terms of the contribution to the luminescence from various excited states. Based on our model, we have made further predictions for the dependence of the DOP on the structure parameters and experimental conditions.

\section{Acknowledgments}

The authors would like to thank the group from the Department of Applied Physics, University of Würzburg (Alfred Forchel, Sven Höfling and Sebastian Hein) for providing the structures used in the experimental part of the study. The work has been supported by the Polish MNiSW (grant No. N N202 181238). A. Musiał acknowledges financial support within a fellowship co-financed by the European Union within the European Social Fund.

\section{References}

[1] T. Utzmeier, P.A. Postigo, J. Tamayo, R. Garcia, F. Briones, Appl. Phys. Lett. 69, 2674 (1996).

[2] A. Sauerwald, T. Kummell, G. Bacher, A. Somers, R. Schwertberger, J.P. Reithmaier, A. Forchel, Appl. Phys. Lett. 86, 253112 (2005).

[3] A. Löffler, J. Reithmaier, A. Forchel, J. Cryst. Growth 286, 6 (2006)

[4] W. Rudno-Rudzinski, R. Kudrawiec, P. Podemski, G. Sek, J. Misiewicz, A. Somers, R. Schwertberger, J.P. Reithmaier, A. Forchel, Appl. Phys. Lett. 89, 031908 (2006).

[5] S. Hein, V. von Hinten, S. Höfling, A. Forchel, Appl. Phys. Lett. 92, 011120 (2008).

[6] G. Sęk, P. Podemski, A. Musiał, J. Misiewicz, S. Hein, S. Hö̈ling, A. Forchel, J. Appl. Phys. 105, 086104 (2009).

[7] H. Dery, E. Benisty, A. Epstein, R. Alizon, V. Mikhelashvili, G. Eisenstein, R. Schwertberger, D. Gold, J.P. Reithmaier, A. Forchel, J. Appl. Phys. 95, 6103 (2004).

[8] M. Jo, T. Mano, K. Sakoda, Appl. Phys. Express 3, 045502 (2010).

[9] J.H. Wei, K.S. Chana, J. Appl. Phys. 97, 123524 (2005).

[10] J. Planelles, M. Royo, A. Ballester, M. Pi, Phys. Rev. B 80, 1 (2009).

[11] P. Miska, J. Even, C. Platz, B. Salem, T. Benyattou, C. Bru-Chevalier, G. Guillot, G. Bremond, K. Moumanis, F.H. Julien, O. Marty, C. Monat, M. Gendry, J. Appl. Phys. 95, 1074 (2004).

[12] J. Andrzejewski, G. Sęk, E. O'Reilly, A. Fiore, J. Misiewicz, J. Appl. Phys. 107, 073509 (2010).

[13] A.V. Koudinov, I A. Akimov, Yu.G. Kusrayev, F. Henneberger, Phys. Rev. B 70, R241305 (2004).

[14] J.J. Sakurai, Modern Quantum Mechanics, Addison Wesley, Reading (MA) 1994.

[15] R. Winkler, Spin-Orbit Coupling Effects in Two-Dimensional Electron and Hole Systems, Vol. 191 of Springer Tracts in Modern Physics, Springer, Berlin 2003.

[16] C. Pryor, Phys. Rev. B 57, 7190 (1998). 